\title{
STABILITY FOR AN INVERSE PROBLEM IN POTENTIAL THEORY
}

\author{
HAMID BELLOUT, AVNER FRIEDMAN AND VICTOR ISAKOV
}

\begin{abstract}
Let $D$ be a subdomain of a bounded domain $\Omega$ in $\mathbb{R}^{n}$. The conductivity coefficient of $D$ is a positive constant $k \neq 1$ and the conductivity of $\Omega \backslash D$ is equal to 1 . For a given current density $g$ on $\partial \Omega$, we compute the resulting potential $u$ and denote by $f$ the value of $u$ on $\partial \Omega$. The general inverse problem is to estimate the location of $D$ from the known measurements of the voltage $f$. If $D_{h}$ is a family of domains for which the Hausdorff distance $d\left(D, D_{h}\right)$ equal to $O(h)$ ( $h$ small), then the corresponding measurements $f_{h}$ are $O(h)$ close to $f$. This paper is concerned with proving the inverse, that is, $d\left(D, D_{h}\right) \leq \frac{1}{c}\left\|f_{h}-f\right\|, c>0$; the domains $D$ and $D_{h}$ are assumed to be piecewise smooth. If $n \geq 3$, we assume in proving the above result, that $D_{h} \supset D$ (or $D_{h} \subset D$ ) for all small $h$. For $n=2$ this monotonicity condition is dropped, provided $g$ is appropriately chosen. The above stability estimate provides quantitative information on the location of $D_{h}$ by means of $f_{h}$.
\end{abstract}

\section{INTRODUCTION}

For any two domains $D_{1}, D_{2}$ in $\mathbb{R}^{n}$ denote by $d\left(D_{1}, D_{2}\right)$ the Hausdorff distance between them. Let $\Omega$ be a bounded domain in $\mathbb{R}^{n}(n \geq 2)$ and let $D$ and $D_{h}$ (for any $0<h<h_{0}, h_{0}$ small) be subdomains of $\Omega$ with closure in $\Omega$ such that

$$
c_{1} h \leq d\left(D, D_{h}\right) \leq c_{2} h
$$

where $c_{1}, c_{2}$ are positive constants. Set

$$
a_{D}=\left\{\begin{array}{ll}
k & \text { in } D, \\
1 & \text { in } \Omega \backslash D,
\end{array} \quad a_{D_{h}}= \begin{cases}k & \text { in } D_{h}, \\
1 & \text { in } \Omega \backslash D_{h},\end{cases}\right.
$$

where $k$ is a positive constant, $k \neq 1$. Consider the Neumann problems

$$
\operatorname{div}\left(a_{D} \nabla u_{D}\right)=0 \text { in } \Omega \text {, }
$$

$$
\frac{\partial u_{D}}{\partial \nu}=g \quad \text { on } \partial \Omega, \quad \int_{\Omega} u_{D}=0
$$

and

$$
\begin{array}{cl}
\operatorname{div}\left(a_{D_{h}} \nabla u_{D_{h}}\right)=0 & \text { in } \Omega, \\
\frac{\partial u_{D_{h}}}{\partial \nu}=g \quad \text { on } \partial \Omega, & \int_{\Omega} u_{D_{h}}=0,
\end{array}
$$

Received by the editors April 9, 1990.

1980 Mathematics Subject Classification (1985 Revision). Primary 86A20; Secondary 58G10, $35 \mathrm{~J} 25$.

The second author's research is partially supported by the National Science Foundation grant DMS-86-12880. 
where $g$ is a given function, satisfying

$$
g \in L^{2}(\partial \Omega), \quad g \neq 0, \quad \int_{\partial \Omega} g=0
$$

and set

$$
f=\left.u_{D}\right|_{\partial \Omega}, \quad f_{h}=\left.u_{D_{h}}\right|_{\partial \Omega} .
$$

We are interested in establishing a local stability estimate of the form

$$
d\left(D, D_{h}\right) \leq C\left|f-f_{h}\right|_{L^{\prime}(\Gamma)}
$$

where $\Gamma$ is a nonempty open subset of $\partial \Omega$, and $h$ is sufficiently small. Such an estimate means that the mapping

$$
\left.D \rightarrow \mathscr{F}(D) \equiv u_{D}\right|_{\Gamma}
$$

has nonzero "derivative."

We shall refer to the case

$$
D_{h} \supset D \text { for all } h\left(\text { or } D_{h} \subset D \text { for all } h\right. \text { ) }
$$

as the monotone case. When the assumption (1.6) is dropped, we speak of the nonmonotone case.

We shall always assume that $\partial D$ is piecewise smooth, and that $\partial D_{h}$ has the representation

$$
\partial D_{h}: x=f(s)+h \sigma_{h}(s) \nu(s) \text { a.e. }
$$

where $\nu(s)$ is the normal to $\partial D$, wherever it exists, and $\left|\sigma_{h}(s)\right| \leq C ; s$ is an $(n-1)$-dimensional local parameter. Notice that $\partial D$ is given by $x=f(s)$.

Bellout and Friedman [1] established (1.5) in the monotone case, provided $\partial D$ and $\partial D_{h}$ are in $C^{2, \alpha}$ (uniformly in $h$ ); their proof actually requires only $C^{1,1}$ smoothness. An earlier proof of (1.5) for $n=2$, due to Friedman and Gustafsson [5], also required the same smoothness.

For $n=2$ Bellout and Friedman [1] have established (1.5) for the nonmonotone case provided $\partial D$ is analytic and certain finite number of "orthogonality" conditions are satisfied; it is however not easy to verify such conditions even, for instance, if the $D_{h}$ are translates of $D$.

In $\S 4$ we shall extend the stability result (1.5) of Bellout and Friedman to the monotone case when $\partial D$ is only piecewise $C^{1,1}$; the proof requires some new ideas and technical estimates which are developed in $\S \S 2,3$. Our interest in the piecewise $C^{1,1}$ case and in particular in polyhedra stems from a recent uniqueness theorem due to Friedman and Isakov [6]. They proved that if $D$ and $D^{\prime}$ are any convex polyhedra in $\Omega$ such that the solution $u_{D}$ of $(1.1)$ and the corresponding solution $u_{D^{\prime}}$ for $D^{\prime}$ satisfy: $u_{D}=u_{D^{\prime}}$ on an open nonempty portion $\Gamma$ of $\partial \Omega$, then $D=D^{\prime}$. They needed to assume that either $\Omega$ is a half-space or $D$ and $D^{\prime}$ are not "too close" to $\partial \Omega$. They also established (1.5), but only when $\Omega$ is a half-space and under some severe restrictions on $D$.

In $\S 5$ we consider the case $n=2$ and $\partial D$ analytic, but drop the monotonicity assumption (1.6). We establish the stability estimate (1.5) for appropriately chosen function $g$. 
Finally in $\S 6$ we extend the results of $\S 5$ to the case where $D$ is a convex polygon or, more generally, piecewise analytic.

\section{THE BeHAVIOR OF $\nabla u$ NEAR A VERTEX OF $\partial D$}

Throughout this paper we assume that $\Omega$ is a bounded domain in $\mathbb{R}^{n} \quad(n \geq 2)$ with $C^{1, \alpha}$ boundary.

Let $D$ be a subdomain of $\Omega$ with $\bar{D} \subset \Omega$ and let

$$
a(x)= \begin{cases}k & \text { if } x \in D, \\ 1 & \text { if } x \in \Omega \backslash D,\end{cases}
$$

where $k$ is a positive number $\neq 1$. Consider the diffraction problem

$$
\begin{gathered}
\operatorname{div}(a \nabla u)=f \quad \text { in } \Omega, \\
\frac{\partial u}{\partial \nu}=g \quad \text { on } \partial \Omega, \quad \int_{\Omega} u=0,
\end{gathered}
$$

where $g$ satisfies

$$
\begin{gathered}
g \in L^{2}(\partial \Omega), \quad g \neq 0, \\
\int_{\partial \Omega} g=\int_{\Omega} f .
\end{gathered}
$$

We shall be interested in the behavior of $\nabla u$ near a point $x_{0} \in \partial D$ where $\partial D$ is not smooth. For simplicity we first consider the case when $n=2, x_{0}=0$ and, for some ball $B_{r_{0}} \equiv B_{r_{0}}\left(x_{0}\right)$,

$$
\begin{gathered}
\partial D \cap \bar{B}_{r_{0}} \quad \text { consists of line segments } \\
l_{1}=\left\{(r, \theta) ; 0 \leq r \leq r_{0}, \theta=0\right\}, \quad \text { and } \\
l_{2}=\left\{(r, \theta) ; 0 \leq r \leq r_{0}, \theta=\beta\right\}, \quad 0<\beta<\pi .
\end{gathered}
$$

Consider first the case $f=0$ and set $u^{e}=u_{\Omega \backslash D}, u^{i}=u_{D}$.

Lemma 2.1. The following expansion holds for $0<r<r_{1} \quad\left(r_{1}=r_{0} / 2\right)$ :

$$
\begin{aligned}
& u^{e}=u^{e}(0)+\sum_{j=1}^{\infty} r^{\gamma_{j}}\left(A_{j}^{e} \cos \gamma_{j} \theta+B_{j}^{e} \sin \gamma_{j} \theta\right), \\
& u^{i}=u^{i}(0)+\sum_{j=1}^{\infty} r^{\gamma_{j}}\left(A_{j}^{i} \cos \gamma_{j} \theta+B_{j}^{i} \sin \gamma_{j} \theta\right) ;
\end{aligned}
$$

the series are convergent with their first derivatives, absolutely and uniformly for $0<r \leq r_{1}$. Here, the sequence $\gamma_{j}$ is monotone increasing,

$$
0<c_{1} \leq \gamma_{j} / j \leq c_{2}<\infty \text { for all } j
$$

and

$$
\gamma_{1}>\frac{1}{2}
$$

Proof. Denote by $S^{1}$ the unit circle and define on $S^{1}$

$$
a(\theta)= \begin{cases}k & \text { if } 0 \leq \theta \leq \beta, \\ 1 & \text { if } \beta<\theta<2 \pi .\end{cases}
$$


Introduce the function spaces $L_{a}^{2}\left(S^{1}\right), H_{a}^{1}\left(S^{1}\right)$ with norms

$$
\begin{gathered}
\|v\|_{L_{a}^{2}\left(S^{1}\right)}=\left\{\int_{0}^{2 \pi} a|v(\theta)|^{2} d \theta\right\}^{1 / 2}, \\
\|v\|_{H_{a}^{1}\left(S^{1}\right)}=\left\{\int_{0}^{2 \pi} a v_{\theta}^{2}(\theta) d \theta+\int_{0}^{2 \pi} a v^{2}(\theta) d \theta\right\}^{1 / 2} .
\end{gathered}
$$

Set

$$
\mathscr{L} v=\frac{1}{a} \frac{\partial}{\partial \theta}\left(a \frac{\partial}{\partial \theta} v\right)
$$

$\mathscr{L}$ is an unbounded, selfadjoint, positive elliptic operator with dense domain in $L_{a}^{2}\left(S^{1}\right)$, and $(\mathscr{L}+1)^{-1}$ is compact. Hence the spectrum of $\mathscr{L}$ consists of positive eigenvalues $\gamma_{j}^{2} \quad\left(\gamma_{j}>0\right)$. We denote a corresponding (complete) orthonormal sequence by $\left\{v_{j}\right\}$; it is a basis for $L_{a}^{2}\left(S^{1}\right)$.

If

$$
\mathscr{L} v+\gamma_{j}^{2} v=0
$$

then

$$
v^{\prime \prime}+\gamma_{j}^{2} v=0 \text { on } 0<\theta<\beta \text { and on } \beta<\theta<2 \pi,
$$

so that

$$
\begin{array}{cl}
v_{j}=v_{j}^{i}=M_{j}^{i} \cos \gamma_{j} \theta+N_{j}^{i} \sin \gamma_{j} \theta, & 0<\theta<\beta, \\
v_{j}=v_{j}^{e}=M_{j}^{e} \cos \gamma_{j} \theta+N_{j}^{e} \sin \gamma_{j} \theta, & \beta<\theta<2 \pi ;
\end{array}
$$

in addition, the diffraction (or transmission) conditions

$$
\left.\begin{array}{c}
v_{j}^{i}=v_{j}^{e} \\
k v_{j, \theta}^{i}=v_{j, \theta}^{e}
\end{array}\right\} \quad \text { at } \theta=0, \theta=\beta
$$

must be satisfied, as well as the condition

$$
\int_{0}^{2 \pi} v_{j}^{2} d \theta=1
$$

For every $r \in\left(0, r_{0}\right)$ we have the expansion

$$
u(r, \theta)=u(0)+\sum_{j=1}^{\infty} h_{j}(r) v_{j}(\theta) \text { in } L^{2}\left(S^{1}\right)
$$

and

$$
h_{j}(r)=\int_{0}^{2 \pi} a u v_{j} d \theta .
$$

The function $u$ satisfies, for $0<r<r_{0}$,

$$
a\left(\frac{1}{r}\left(r u_{r}\right)_{r}\right)+\frac{1}{r^{2}}\left(a u_{\theta}\right)_{\theta}=0 .
$$

Multiplying (2.15) by $a v_{j}(\theta)$ and integrating over $S^{1}$ we get, after using (2.10),

$$
\frac{1}{r}\left[r\left(\int_{S^{1}} a u v_{j} d \theta\right)_{r}\right]_{r}-\frac{\gamma_{j}^{2}}{r^{2}} \int_{S^{1}} a u v_{j}=0 .
$$


Hence $h_{j}(r)$ satisfies

$$
\frac{1}{r}\left(r h_{j, r}\right)_{r}-\frac{\gamma_{j}^{2}}{r^{2}} h_{j}=0
$$

so that

$$
h_{j}(v)=C_{j} r^{\gamma_{j}}+D_{j} r^{-\gamma_{j}} .
$$

Since $u_{r} \in L^{2}\left(B_{r_{0}}\right)$ we have

$$
\int_{0}^{r_{0}}\left[\left(u v_{j}\right)_{r}\right]^{2} r d r \leq M<\infty
$$

i.e.,

$$
\int_{0}^{r_{0}}\left|h_{j}^{\prime}(r)\right|^{2} r d r \leq M .
$$

It follows that $D_{j}=0$ and, consequently, from (2.14),

$$
u(r, \theta)=u(0)+\sum C_{j} r^{\gamma_{j}} v_{j}(\theta)
$$

and

$$
\int_{S^{1}} a u^{2}(r, \theta) d \theta=u^{2}(0)+\sum C_{j}^{2} r^{2 \gamma_{j}} \quad\left(0<r \leq r_{0}\right) .
$$

Since $u_{r} \in L_{a}^{2}\left(S^{1}\right)$ for $r=r_{0}$, we actually even have

$$
\int_{S^{1}} a u_{r}^{2}(r, \theta) d \theta=\sum C_{j}^{2} \gamma_{j}^{2} r^{2\left(\gamma_{j}-1\right)}<\infty
$$

for $r=r_{0}$, so that

$$
\sum_{j=1}^{\infty} C_{j}^{2} \gamma_{j}^{2} r_{0}^{2 \gamma_{j}}<\infty
$$

We next estimate the $\gamma_{j}$. We can write

$$
v_{j}^{e}=\operatorname{Re}\left\{a_{j} e^{i \gamma_{j} \theta}\right\}, \quad v_{j}^{i}=\operatorname{Re}\left\{b_{j} e^{i \gamma_{j} \theta}\right\} .
$$

The refraction conditions at $\theta=0$ and $\theta=\beta$ then become (for $a=a_{j}, b=$ $\left.b_{j}, \gamma=\gamma_{j}\right)$

$$
\begin{gathered}
a+\bar{a}=b e^{i \gamma 2 \pi}+\bar{b} e^{-i \gamma 2 \pi} \\
a e^{i \gamma \beta}+\bar{a} e^{-i \gamma \beta}=b e^{i \gamma \beta}+\bar{b} e^{-i \gamma \beta}
\end{gathered}
$$

and

$$
\begin{gathered}
k(a-\bar{a})=b e^{i \gamma 2 \pi}-\bar{b} e^{-i \gamma 2 \pi}, \\
k\left(a e^{i \gamma \beta}-\bar{a} e^{-i \gamma \beta}\right)=b e^{i \gamma \beta}-\bar{b} e^{-i \gamma \beta} .
\end{gathered}
$$

Taking $k$ times (2.23) and adding to (2.25), we get

$$
2 k a=(k+1) b e^{i \gamma 2 \pi}+(k-1) \bar{b} e^{-i \gamma 2 \pi} .
$$

Similarly, taking $k$ times (2.24) and adding to (2.26), we get

$$
2 k a=(k+1) b+(k-1) \bar{b} e^{-2 i \gamma \beta} \text {. }
$$


Comparing (2.27) with (2.28) we find that

$$
(k+1) b\left(e^{i \gamma 2 \pi}-1\right)=(k-1) \bar{b}\left(e^{-2 i \gamma \beta}-e^{-2 i \gamma \pi}\right) .
$$

We need to consider two cases

Then

Case (i). $e^{i i \gamma \pi} \neq 1$.

$$
\frac{b}{\bar{b}}=\frac{k-1}{k+1} \frac{e^{-2 i \gamma \beta}-e^{-2 i \gamma \pi}}{e^{2 i \gamma \pi}-1} .
$$

Since $|b / \bar{b}|=1$, we conclude that

$$
\frac{\left|e^{-2 i \gamma \beta}-e^{-2 i \gamma \pi}\right|}{\left|e^{2 i \gamma \pi}-1\right|}=\left|\frac{k+1}{k-1}\right| \equiv A>1,
$$

or

$$
\sin \gamma(\pi-\beta)=A \sin \gamma \pi
$$

and it is easy to see that this equation has an infinite sequence of solutions $\gamma_{j}$ satisfying (2.7). We claim that the smallest one, $\gamma_{1}$, satisfies $\gamma_{1}>\frac{1}{2}$. Indeed, if $\gamma_{1} \leq \frac{1}{2}$ then $2 \pi \gamma_{1}<\pi$ and $0<2 \gamma_{1} \beta<2 \pi \gamma_{1}<\pi$. But then

$$
\left|e^{2 i \gamma_{1} \pi}-e^{2 i \gamma_{1} \beta}\right|<\left|e^{2 i \gamma_{1} \pi}-1\right|,
$$

a contradiction to $(2.30)$.

Case (ii). $e^{2 i \gamma \pi}=1$.

Then $\gamma=\gamma_{j}=n$ for some integer $n$, and from (2.29) we see that $\beta \gamma / \pi$ is also an integer; consequently

$$
\beta=\frac{q}{m} \pi, \quad q \text { and } m \text { are relatively prime positive integers. }
$$

We easily see that all the additional solutions $\gamma$, in this case, are multiples of $m$. Thus the asserted expansion (2.14) still holds, but one has to include the additional sequence of multiples of $m$ into the sequence of the $\gamma_{j}$ 's.

Finally, using (2.22) it is easily seen that the series expansion of $u(r, \theta)$ and its gradient are absolutely uniformly convergent for $0<r \leq r_{0} / 2$.

We shall now extend Lemma 2.1 to the case $f \not \equiv 0$, assuming that $f \in$ $L^{4 / 3}(\Omega)$.

Set

$$
f_{j}(r)=\int_{0}^{2 \pi} f(r, \theta) v_{j}(\theta) d \theta
$$

Then formally

$$
u(r, \theta)=u(0)+\sum C_{j} r^{\gamma_{j}} v_{j}(\theta)+\sum e_{j}(r) v_{j}(\theta)
$$

where

$$
e_{j}(r)=\frac{r^{\gamma_{j}}}{2 \gamma_{j}} \int_{r_{0} / 2}^{r} f_{j}(s) s^{1-\gamma_{j}} d s-\frac{r^{-\gamma_{j}}}{2 \gamma_{j}} \int_{0}^{r} f_{j}(s) s^{1+\gamma_{j}} d s
$$

is a solution of

$$
\frac{1}{r}\left(r h^{\prime}\right)^{\prime}-\frac{\gamma_{j}^{2}}{r^{2}} h=f_{j}(r)
$$

the fact that $D_{j}=0$ follows by using (2.19) as before, noting that $e_{j}=$ $O\left(r^{2}\right), e_{j}^{\prime}=O(r)$. Observe that the first integral on the right-hand side of (2.34) 
is from $r_{0} / 2$ to $r$ (the integral from 0 to $r$ will not converge if $\gamma_{j} \geq 2$ ). We have,

$$
\begin{aligned}
& \left|\int_{0}^{r} f_{j}(s) s^{1+\gamma_{j}} d s\right|=\left|\int_{0}^{r} \int_{0}^{2 \pi} f(s, \theta) v_{j}(\theta) s^{1 / p} s^{1+\gamma_{j}} s^{1-1 / p} d \theta d s\right| \\
& \quad \leq\left(\int_{0}^{r} \int_{0}^{2 \pi}|f|^{p} s d \theta d s\right)^{1 / p}\left(\int_{0}^{r} \int_{0}^{2 \pi} s^{1+\left(1+\gamma_{j}\right) q}\left|v_{j}(\theta)\right|^{q}\right)^{1 / q} \quad\left(q=\frac{p}{p-1}\right) \\
& \quad \leq \frac{C r^{1+\gamma_{j}+1 / q}}{\gamma_{j}^{1 / q}}\left(\int\left|v_{j}(\theta)\right|^{q}\right)^{1 / q},
\end{aligned}
$$

if $p=\frac{4}{3}, q=4$ (since $\left.f \in L^{4 / 3}\right)$. Noting that by Sobolev's imbedding [4, p. 27],

$$
\left|v_{j}\right|_{L^{3}} \leq C\left(\left|v_{j}^{\prime}\right|_{L^{2}}\right)^{a}\left(\left|v_{j}\right|_{L^{2}}\right)^{1-a}, \quad a=\frac{1}{4}
$$

and

$$
\left|v_{j}\right|_{L^{2}}=1, \quad\left|v_{j}^{\prime}\right|_{L^{2}} \leq C \gamma_{j}\left|v_{j}\right|_{L^{2}}=C \gamma_{j},
$$

we get

$$
\left|v_{j}\right|_{L^{3}} \leq C \gamma_{j}^{1 / 4}
$$

Substituting this into (2.35), we get

$$
\left|\int_{0}^{r} f_{j}(s) s^{1+\gamma_{j}} d s\right| \leq \frac{C r^{1+\gamma_{j}+1 / 4}}{\gamma_{j}^{1 / 4}}
$$

A similar estimate holds for the second integral in (2.34). Hence

$$
\sum\left|e_{j}(r) v_{j}(\theta)\right| \leq \sum \frac{c r^{2}}{\gamma_{j}^{1+1 / 4}} \leq C r^{2}
$$

by $(2.7)$.

From (2.33), (2.36) we deduce that the series

$$
\sum_{j} C_{j} r^{\gamma_{j}} v_{j}(\theta)
$$

is convergent in $L^{2}\left(S^{1}\right)$ and therefore

$$
\sum C_{j}^{2} r^{2 \gamma_{j}}<\infty, \quad 0<r<r_{0} .
$$

This implies the absolute uniform convergence of the series $(2.37)$ for $0<r \leq$ $r_{0} / 2$; in particular,

$$
|u(r, \theta)-u(0)| \leq C r^{\gamma_{1}}, \quad \gamma_{1}>\frac{1}{2} .
$$

We now consider the function

$$
v_{\lambda}(x)=u(\lambda x)-u(0)
$$

for $\lambda$ small and $x$ in $B_{*}=\left\{\frac{1}{4}<|x|<4\right\}$. Let $B_{0}=\left\{\frac{1}{2}<|x|<\frac{7}{2}\right\}$. Clearly

$$
\operatorname{div}\left(a \nabla v_{\lambda}\right)=\lambda^{2} f(\lambda x), \quad\left|v_{\lambda}\right| \leq C \lambda^{\gamma_{1}}
$$


Let $l_{\lambda}$ be any line in $\widetilde{B}=\{1<|x|<3\}$ with endpoints on $\partial \widetilde{B}$. Then, by the trace imbedding (of $H^{1 / 2}(B) \rightarrow L^{2}\left(l_{\lambda}\right)$ ), Sobolev's imbedding [7, p. 27] and $L^{p}$ elliptic estimates,

$$
\left\{\int_{l_{\lambda}}\left|\nabla v_{\lambda}\right|^{2} d x\right\}^{1 / 2} \leq C\left|v_{\lambda}\right|_{W^{2,4 / 3}\left(B_{0}\right)} \leq C \int_{B_{*}}\left|\lambda^{2} f(\lambda x)\right|^{4 / 3} d x+C_{1} \lambda^{\gamma_{1}} .
$$

Making the substitution $\lambda x=y$ we find that

$$
\int_{l}|\nabla u|^{2} \leq C\left(\int_{\lambda / 4<|x|<\lambda}|f|^{4 / 3}\right)^{3 / 2}+C \lambda^{\gamma_{1}-1 / 2},
$$

where $l$ is the image of $l_{\lambda} ; l$ is any interval connecting a point on $\{r=1 / \lambda\}$ to a point on $\{r=3 / \lambda\}$. By varying $\lambda$, taking for instance $\lambda=3^{-j}$, we deduce that

$$
\int_{l}|\nabla u|^{2} \leq C \varepsilon(|l|), \quad \varepsilon(t) \downarrow 0 \text { if } t \downarrow 0
$$

where $l$ is an interval in $\left\{r<\varepsilon_{0}\right\}$ and $|l|=$ length of $l$.

If $D$ is a polygon, then by applying the above estimate near each vertex of $\partial D$ we arrive at the following result:

Lemma 2.2. Suppose $D$ is a polygon and $f \in L^{4 / 3}(\Omega)$. Then the solution of the refraction problem (2.1), (2.2) satisfies:

For any family of intervals which are the intersection of straight lines parallel to one of the sides of $D$ and $\Omega_{0}$, a compact subset of $\Omega$,

$$
\|\nabla u\|_{L^{2}(l)} \leq C\|f\|_{L^{4 / 3}(\Omega)}
$$

where $C$ is a constant depending only on $D, \Omega_{0}$ and $g$; furthermore, for any vertex $a$ of $\partial D$,

$$
\|\nabla u\|_{L^{2}(\ln B(a, r))} \leq C \varepsilon(|l|), \quad \varepsilon(t) \downarrow 0 \text { if } t \downarrow 0 .
$$

Extension of this result to piecewise smooth domain in any number of dimension will be discussed in $\S 4$.

\section{AN AUXILIARY ESTIMATE}

Let $D$ be a polygon in $\mathbb{R}^{2}$ with edges $\Gamma_{1}, \Gamma_{2}, \ldots, \Gamma_{N}$ and vertices $s_{1}, s_{2}$, $\ldots, s_{N}$ such that $s_{j}=\bar{\Gamma}_{j} \cap \bar{\Gamma}_{j+1}, \Gamma_{N+1}=\Gamma_{1}$. Let $D_{h} \quad\left(0<h \leq h_{0}\right)$ be a family of polygons with edges $\Gamma_{1}(h), \Gamma_{2}(h), \ldots, \Gamma_{N}(h)$ and vertices $s_{1}(h), \ldots, s_{N}(h)$ such that $s_{j}(h)=\overline{\Gamma_{j}(h)} \cap \overline{\Gamma_{j+1}(h)}, \Gamma_{N+1}(h)=\Gamma_{1}(h)$. We assume that

$$
\begin{gathered}
D \subset D_{h}, \quad \bar{D}_{h} \subset \Omega \quad \text { for } 0<h \leq h_{0}, \\
c_{1} h \leq \sum_{j=1}^{N}\left|s_{j}(h)-s_{j}\right| \leq c_{2} h \quad\left(0<c_{1}<c_{2}<\infty\right) .
\end{gathered}
$$

Set

$$
a=\left\{\begin{array}{ll}
k & \text { in } D, \\
1 & \text { in } \Omega \backslash D,
\end{array} \quad a_{h}=\left\{\begin{array}{ll}
k & \text { in } D_{h} \\
1 & \text { in } \Omega \backslash D_{h}
\end{array} \quad(k>0, k \neq 1),\right.\right.
$$


and consider the diffraction problems

$$
\begin{array}{cc}
\operatorname{div}(a \nabla u)=0 & \text { in } \Omega, \\
\frac{\partial u}{\partial \nu}=g \quad \text { on } \partial \Omega, & \int_{\Omega} u=0
\end{array}
$$

and

$$
\begin{array}{cc}
\operatorname{div}\left(a_{h} \nabla u_{h}\right)=0 & \text { in } \Omega, \\
\frac{\partial u_{h}}{\partial \nu}=g \quad \text { on } \partial \Omega, & \int_{\Omega} u_{h}=0
\end{array}
$$

where $g$ satisfies

$$
g \in L^{2}(\Omega), \quad g \neq 0, \quad \int_{\partial \Omega} g=0 .
$$

We are interested in estimating the "quotient difference"

$$
U_{h}=\frac{u_{h}-u}{h} \text {. }
$$

Lemma 3.1. For any $0<\varepsilon<2$ there is a constant $C$ such that

$$
\int_{\Omega}\left|U_{h}\right|^{2+\varepsilon} \leq C \quad \forall 0<h \leq h_{0} .
$$

For $\varepsilon=0$ and $\partial D \in C^{1,1}$ this was proven by Bellout and Friedman [1].

Proof. Multiplying the difference of the equations (3.2), (3.4) by a function $v$ in $H^{1}(\Omega)$ and integrating over $\Omega$, we easily get

$$
\int_{\Omega} a_{h} \nabla U_{h} \cdot \nabla v+\frac{k-1}{h} \int_{D_{h} \backslash D} \nabla u \cdot \nabla v=0 \quad \forall v \in H^{1}(\Omega) .
$$

We introduce the solution $w_{h}$ to the diffraction problem

$$
\begin{array}{cl}
\operatorname{div}\left(a_{h} \nabla w_{h}\right)=U_{h} & \text { in } \Omega, \\
\frac{\partial w_{h}}{\partial \nu}=0 \quad \text { on } \partial \Omega, \quad & \int_{\Omega} w_{h}=0 ;
\end{array}
$$

since $\int_{\Omega} U_{h}=0$, this problem does in fact have a unique solution.

Multiplying (3.10) by $U_{h}$ and integrating over $\Omega$, we get

$$
-\int_{\Omega} a_{h} \nabla w_{h} \cdot \nabla U_{h}=\int_{\Omega} U_{h}^{2}
$$

Substituting $v=w_{h}$ in (3.9) and adding the result to (3.12), we find that

$$
\frac{k-1}{h} \int_{D_{h} \backslash D} \nabla u \cdot \nabla w_{h}=\int_{\Omega} U_{h}^{2},
$$

so that, by Cauchy's inequality,

$$
\int_{\Omega} U_{h}^{2} \leq \frac{|k-1|}{h}\left\{\int_{D_{h} \backslash D}|\nabla u|^{2}\right\}^{1 / 2}\left\{\int_{D_{h} \backslash D}\left|\nabla w_{h}\right|^{2}\right\}^{1 / 2} .
$$


By extending $\Gamma_{j}$ at $s_{j+1}$ as a line segment until it meets $\partial D_{h}$, for $1 \leq j \leq N$, we get a "triangulation" of $D_{h} \backslash D$ into $N$ quadrangles $Q_{j}(h)$, each bounded by the extended $\Gamma_{j-1}, \Gamma_{j}$ and portions of $\Gamma_{j}(h), \Gamma_{j+1}(h)$. Each $Q_{j}(h)$ can be traced by a family of intervals $l_{j}(\lambda, h)$ parallel to $\Gamma_{j}$ at distance $\lambda$ from $\Gamma$, where $\lambda$ varies in some interval $0 \leq \lambda \leq h_{j}, h_{j} \leq C h$. Hence

$$
\begin{aligned}
\int_{D_{h} \backslash D}|\nabla u|^{2} & \leq \sum_{j=1}^{N} \int_{Q_{j}(h)}|\nabla u|^{2}=\sum_{j=1}^{N} \int_{0}^{h_{j}} d \lambda \int_{l_{j}(\lambda, h)}|\nabla u|^{2} \\
& \leq \sum_{j=1}^{N} \int_{0}^{h_{j}} C d \lambda, \quad \text { by Lemma 2.2. }
\end{aligned}
$$

It follows that

$$
\int_{D_{h} \backslash D}|\nabla u|^{2} \leq C h
$$

Similarly

$$
\int_{D_{h} \backslash D}\left|\nabla w_{h}\right|^{2} \leq \sum_{j=1}^{N} \int_{0}^{h_{j}} d \lambda \int_{l_{j}(\lambda, h)}\left|\nabla w_{h}\right|^{2}
$$

and

$$
\int_{l_{j}(\lambda, h)}\left|\nabla w_{h}\right|^{2} \leq C \int_{\Omega}\left|U_{h}\right|^{2},
$$

by Lemma 2.2 applied to $w_{h} /\left\{\int_{\Omega}\left|U_{h}\right|^{2}\right\}^{1 / 2}$; hence

$$
\int_{D_{h} \backslash D}\left|\nabla w_{h}\right|^{2} \leq C h \int_{\Omega} U_{h}^{2}
$$

Substituting the estimates (3.14), (3.15) into the right-hand side of (3.13), we conclude that

$$
\int_{\Omega} U_{h}^{2} \leq C\left\{\int_{\Omega} U_{h}^{2}\right\}^{1 / 2}
$$

i.e.,

$$
\int_{\Omega} U_{h}^{2} \leq C
$$

Having proved (3.8) for $\varepsilon=0$ we proceed to prove it for $\varepsilon$ positive and small. For this purpose we introduce another auxiliary function $w_{h}$ defined as the solution to

$$
\operatorname{div}\left(a_{h} \nabla w_{h}\right)=\left|U_{h}\right|^{\varepsilon} U_{h}-A \equiv F_{\varepsilon} \quad \text { in } \Omega
$$

with the same conditions (3.11) as before; the constant $A$ is chosen so that $F_{\varepsilon}$ satisfies the compatibility condition $\int_{\Omega} F_{\varepsilon}=0$, that is

$$
A=\frac{1}{|\Omega|} \int_{\Omega}\left|U_{h}\right|^{\varepsilon} U_{h} .
$$

From (3.16) it follows that

$$
\int_{\Omega}\left|F_{\varepsilon}\right|^{4 / 3} \leq C,
$$


if

$$
\frac{4}{3}=\frac{2}{1+\varepsilon}, \quad \text { i.e., if } \varepsilon=\frac{1}{2} \text {. }
$$

We can then apply Lemma 2.2 and deduce that

$$
\int_{l_{j}(\lambda, h)}\left|\nabla w_{h}\right|^{2} \leq C,
$$

for any line $l_{j}(\lambda, h)$ and, consequently,

$$
\int_{D_{h} \mid D}\left|\nabla w_{h}\right|^{2} \leq C h
$$

Next we multiply (3.17) by $U_{h}$ and integrate over $\Omega$. Since $\int_{\Omega} U_{h}=0$, we obtain

$$
-\int_{\Omega} a_{h} \nabla w_{h} \cdot \nabla U_{h}=\int_{\Omega}\left|U_{h}\right|^{2+\varepsilon} .
$$

Substituting $v=w_{h}$ in (3.9) and adding to (3.19), we find that

$$
\int_{\Omega}\left|U_{h}\right|^{2+\varepsilon}=\frac{k-1}{h} \int_{D_{h} \backslash D} \nabla u \cdot \nabla w_{h},
$$

and using the estimates (3.14), (3.18), we get

$$
\int_{\Omega}\left|U_{h}\right|^{2+\varepsilon} \leq C
$$

where $C$ is a constant independent of $h$, or ( since $\varepsilon=1 / 2)$

$$
\int_{\Omega}\left|U_{h}\right|^{2+1 / 2} \leq C
$$

which is an improvement of (3.16). More generally, assuming that (3.8) holds for $\varepsilon=\varepsilon_{m}$ the above proof shows that (3.8) will then hold for $\varepsilon=\varepsilon_{m+1}$ where

$$
\frac{4}{3}=\frac{2+\varepsilon_{m}}{1+\varepsilon_{m+1}}
$$

and since $\varepsilon_{m} \uparrow 2$ if $m \uparrow \infty$, the lemma follows.

\section{Stability in the Monotone case}

For simplicity we begin with the case where $n=2$ and $D, D_{h}$ are polygonal domains as in $\S 3$, satisfying (3.1), and $D$ is convex.

Set

$$
f_{h}=\left.u_{h}\right|_{\partial \Omega}, \quad f=\left.u\right|_{\partial \Omega},
$$

and let $\Gamma$ be a nonempty open subset of $\partial \Omega$.

Theorem 4.1. Under the foregoing assumptions

$$
\liminf _{h \rightarrow 0} \int_{\Gamma} \frac{\left|f_{h}-f\right|}{h}>0
$$

This means that

$$
d\left(D_{h}, D\right) \leq C \int_{\Gamma}\left|f_{h}-f\right|,
$$


where the constant $C$ may depend on the family $\left\{\sigma_{h}\right\}$. We note that the reverse inequality

$$
\int_{\Gamma}\left|f_{h}-f\right| \leq C d\left(D_{h}, D\right)
$$

can easily be established.

From (3.9) we get, by integration by parts,

$$
\begin{gathered}
-\int_{\Omega} a_{h} U_{h} \Delta v+(k-1) \int_{\partial D_{h}} U_{h} \frac{\partial v}{\partial \nu_{e}}+\int_{\partial \Omega} \frac{f_{h}-f}{h} \frac{\partial v}{\partial n} \\
+\frac{k-1}{h} \int_{D_{h} \backslash D} \nabla u \cdot \nabla v=0 \quad \forall v \in H^{2}(\Omega)
\end{gathered}
$$

where $\nu_{e}$ is the exterior normal to $\partial D_{h}$ and $n$ is the exterior normal to $\partial \Omega$.

Suppose (4.2) is not true, i.e., for a sequence $h \rightarrow 0$,

$$
\int_{\Gamma} \frac{\left|f_{h}-f\right|}{h} \rightarrow 0
$$

Since $\Delta U_{h}=0$ in $\Omega \backslash \bar{D}_{h}$ and in $D_{h}, U_{h}$ is uniformly bounded in $L^{2}(\Omega)$ (by Lemma 3.1), we may assume that

$$
U_{h} \rightarrow U \text { uniformly in compact subsets of } \bar{\Omega} \backslash \partial D \text {. }
$$

Since further $U=0$ on $\Gamma$ (by (4.6)) and $\partial U / \partial n=0$ on $\partial \Omega$ we have, by unique continuation of harmonic functions,

$$
U=0 \text { in } \Omega \backslash \bar{D}
$$

also

$$
\Delta U=0 \text { in } D
$$

Consequently

$$
-\int_{\Omega} a_{h} U_{h} \Delta v \rightarrow-\int_{D} k U \Delta v \quad \text { as } h \rightarrow 0 .
$$

We next prove that

$$
\int_{\partial D_{h}} U_{h} \frac{\partial v}{\partial \nu_{e}} \rightarrow 0 \quad \text { as } h \rightarrow 0 .
$$

Since $\partial D_{h}$ consists of $N$ edges $\Gamma_{j}(h)$, it suffices to prove that

$$
\int_{\Gamma_{j}(h)} U_{h} \frac{\partial v}{\partial \nu_{e}} \rightarrow 0
$$

for each $j$.

Let $\sigma_{h}$ be a line segment containing $\overline{\Omega_{j}(h)}$ in its interior and let

$$
F_{h}(x)= \begin{cases}\partial v(x) / \partial \nu_{e} & \text { for } x \in \Gamma_{j}(h), \\ 0 & \text { for } x \in \sigma_{h} \backslash \Gamma_{j}(h) .\end{cases}
$$

Since $F_{h}$ is piecewise smooth, it belongs to $W_{p}^{1-1 / p}\left(\sigma_{h}\right)$ for any $p=2-\delta$, $\delta>0[7$, p. 45]. Hence, by the trace theorem [7, p. 37] there exists a function 
$z_{h}$ defined in semicircle $S_{h}$ in $\Omega \backslash D_{h}$ with diameter $\sigma_{h}$ such that

$$
\begin{gathered}
\left\|z_{h}\right\|_{W^{2, p}\left(S_{h}\right)} \leq C, \\
z_{h}=0 \quad \text { on } \sigma_{h}, \\
\frac{\partial z_{h}}{\partial \nu_{e}}=F_{h} \text { on } \sigma_{h},
\end{gathered}
$$

and

$z_{h}$ vanishes in a neighborhood of $\partial S_{h} \backslash \sigma_{h}$

It follows that

$$
\int_{\Gamma_{j}(h)} U_{h} \frac{\partial v}{\partial \nu_{e}}=\int_{S_{h}} U_{h} \Delta z_{h}
$$

As $h \rightarrow 0, \Gamma_{j}(h) \rightarrow \Gamma_{j}$ and $\sigma_{h} \rightarrow \sigma, S_{h} \rightarrow S$. By regularity of $v, \partial v / \partial \nu_{e}$ on $\sigma_{h}$ converges in $W_{p}^{1-1 / p}$-norm (when the independent variable is properly normalized so as to vary in the same interval $\sigma$, say). By the continuity of lifts (see [7, p. 37]) we then have that $z_{h} \rightarrow z$ in $W_{\text {loc }}^{2, p}(S)$. Recalling Lemma 3.1 we conclude that

$$
\int_{S_{h}} U_{h} \Delta z_{h} \rightarrow \int_{S} U \Delta z
$$

and the right-hand side is equal to zero by (4.8). This completes the proof of (4.11).

Next we observe that, by (4.7),

$$
\int_{\partial \Omega} \frac{f_{h}-f}{h} \frac{\partial v}{\partial n}=\int_{\partial \Omega} U_{h} \frac{\partial v}{\partial n} \rightarrow \int_{\partial \Omega} U \frac{\partial v}{\partial n}=0 .
$$

We finally evaluate the last integral on the left-hand side of (4.5). Let $T_{j}$ be the intersection of $D_{h} \backslash D$ with a square of side $\delta$ centered at the vertex $s_{j}$ of $\partial D$. We can trace $T_{j}$ by two families of intervals $l_{1 j}(\lambda), l_{2 j}(\lambda)$, where the $l_{1 j}(\lambda)$ are parallel to $\Gamma_{j}$ at distance $\lambda$ and the $l_{2 j}(\lambda)$ are parallel to $\Gamma_{j+1}$ at distance $\lambda$. Using (2.4) we get

$$
\begin{aligned}
& \left|\frac{k-1}{h} \int_{T_{j}} \nabla u \cdot \nabla v\right| \\
& \leq \frac{C}{h}\left[\int d \lambda\left\{\int_{l_{i j}(\lambda)}|\nabla u|^{2}\right\}^{1 / 2}+\int d \lambda\left\{\int_{l_{2 j}(x)}|\nabla u|^{2}\right\}^{1 / 2}\right] \\
& \leq \frac{C}{h} h=C \varepsilon(\delta) \rightarrow 0, \quad \varepsilon(\delta) \rightarrow 0 \text { if } \delta \rightarrow 0 .
\end{aligned}
$$

The set $D_{h} \backslash\left(D \cup\left(\bigcup T_{j}\right)\right)$ is a disjoint union of rectangles $Q_{j, \delta}$ with two sides nearly parallel at distance $c(h) h\left(\max c(h)=c_{0}>0\right)$ and the other two sides lying near $s_{j}$ and $s_{j+1}$. Since $u$ is smooth in $\bar{\Omega} \backslash D$ except at the set of points $s_{1}, \ldots, s_{N}$, we deduce that

$$
\frac{1}{h} \int_{Q_{j, \delta}} \nabla u \cdot \nabla v \rightarrow \int_{\Gamma_{j, \delta}} \tilde{\sigma} \nabla u^{e} \cdot \nabla v, \quad \tilde{\sigma} \geq 0
$$


where $\Gamma_{j, \delta} \subset \Gamma_{j}$, and the right-hand side converges to

$$
\int_{\Gamma_{j}} \tilde{\sigma} \nabla u^{e} \cdot \nabla v
$$

as $\delta \rightarrow 0$. Combining this with (4.18) it follows that

$$
\frac{k-1}{h} \int_{D_{h} \backslash D} \nabla u \cdot \nabla v \rightarrow(k-1) \int_{\partial D} \tilde{\sigma} \nabla u^{e} \cdot \nabla v \quad \forall v \in H^{2}(\Omega) .
$$

Notice that $\tilde{\sigma}$ is actually a linear function on each edge $\Gamma_{j}$, and

$$
\tilde{\sigma} \geq 0, \tilde{\sigma} \not \equiv 0 \text { on } \partial D \text {. }
$$

We now take $h \rightarrow 0$ in (4.5) and use (4.19), (4.17), (4.11) and (4.10); we obtain

$$
k \int_{D} U \Delta v=(k-1) \int_{\partial D} \tilde{\sigma} \nabla u^{e} \cdot \nabla v
$$

for any $v \in H^{2}(\Omega)$.

Let $V_{\varepsilon}$ be an $\varepsilon$-neight orhood of $D$. If $v \in H^{2}\left(V_{\varepsilon}\right)$ then we can modify it outside $V_{\varepsilon / 2}$ so as to obtain a function $\tilde{v}$ in $H^{2}(\Omega)$. Since (4.21) is valid for $\tilde{v}$, it is also valid for $v$. Thus (4.21) holds for any $v \in H^{2}\left(V_{\varepsilon}\right)$.

The function $u^{i}=\left.u\right|_{D}$ is smooth in $D$ and therefore for any $x_{0} \in D$ and $0<\lambda<1$, the function

$$
v_{\lambda}(x) \equiv u^{i}\left(x_{0}+\lambda\left(x-x_{0}\right)\right) \quad \text { is in } H^{2}\left(V_{\varepsilon}\right)
$$

for some $\varepsilon>0$. Substituting $v=v_{\lambda}$ into (4.21), we get

$$
\int_{\partial D} \tilde{\sigma}(x) \nabla u^{e}(x) \cdot \nabla u^{i}\left(x_{0}+\lambda\left(x-x_{0}\right)\right)=0 .
$$

Letting $\lambda \uparrow 1$ and using Lemma 2.2, we easily conclude that

$$
\int_{\partial D} \tilde{\sigma}(x) \nabla u^{e}(x) \cdot \nabla u^{i}(x)=0 .
$$

Since finally

$$
k \frac{\partial u^{i}}{\partial \nu_{e}}=\frac{\partial u^{e}}{\partial \nu_{e}}, \quad \frac{\partial u^{i}}{\partial \tau}=\frac{\partial u^{e}}{\partial \tau} \quad \text { on } \partial D
$$

where $\tau$ is the tangential direction, it follows that

$$
\int_{\partial D} \tilde{\sigma}\left|\nabla u^{i}\right|^{2}=0 \text {. }
$$

Recalling (4.20) we deduce that $\nabla u^{i}=0$ on some arc on $\partial D$ and hence, by harmonic continuation, $u=$ const in $\Omega$. This implies, in particular, that $g=\partial u^{e} / \partial n \equiv 0$, which is a contradiction.

As we shall see below, Theorem 4.1 can be extended to general piecewise smooth domains $D, D_{h}$.

Definition 4.1. If each $\Gamma_{j}(h)$ is $C^{1,1}$ curve (instead of a line segment) with $C^{1,1}$-norm bounded independently of $h$, and if the angles $\beta_{j}(h)$ at $s_{j}(h)$ satisfy

$$
0<c_{1} \leq \beta_{j}(h) \leq c_{2}<2 \pi \quad \forall j
$$


then we say that $D_{h}$ is uniformly piecewise $C^{1,1}$. Similarly we define " $D$ is piecewise $C^{1,1}$."

We shall need the following assumptions:

$\left(\mathrm{A}_{1}\right) D_{h}$ are uniformly piecewise $C^{1,1}$ and $D$ is piecewise $C^{1,1}$; further, $D$ is strongly starshaped with the respect to the origin in the sense that $\bar{D} \subset \mu D$ for any $\mu>1$.

$\left(\mathrm{A}_{2}\right)$ The vertices $s_{j}(h)$ of $D_{h}$ and $s_{j}$ of $D$ are such that

$$
\left|s_{j}(h)-s_{j}\right| \leq C h .
$$

$\left(\mathrm{A}_{3}\right)$ The representation (1.7) holds outside some $\delta(h)$-neighborhood of $\left\{s_{1}\right.$, $\left.\ldots, s_{N}\right\}$ where $\delta(h) \rightarrow 0$ if $h \rightarrow 0$; further,

$$
\sigma_{h}(s) \rightarrow \sigma(s) \not \equiv 0 \text { as } h \rightarrow 0
$$

uniformly outside any $\delta_{0}$-neighborhood of $\left\{s_{1}, \ldots, s_{N}\right\}$.

$\left(\mathrm{A}_{4}\right) D_{h} \supset D$ or $D_{h} \subset D \quad \forall 0<h \leq h_{0}$.

Theorem 4.2. Under the assumptions $\left(\mathrm{A}_{1}\right)-\left(\mathrm{A}_{4}\right)$, the stability property (4.2) holds.

The proof is similar to the proof of Theorem 4.1. The main difference occurs in the estimates near a vertex. Here we first perform a local diffeomorphism so as to make $D$ locally a sector, and then proceed as before, with minor changes.

We now proceed to the case of dimension $n \geq 2$.

Definition 4.2. Let $D$ be a domain in $\mathbb{R}^{n}(n \geq 2)$. We shall say that $D$ is piecewise $C^{1,1}$ if for any $x_{0} \in \partial D$ there exists a polyhedron $D_{*}$ in $\mathbb{R}^{n}$, a point $x_{*} \in \partial D_{*}$ and a $C^{1,1}$ diffeomorphism $G_{x_{0}}$ from a ball $B\left(x_{0}, \delta\right)$ onto a ball $B\left(x_{*}, \delta_{*}\right)$ such that

$$
G_{x_{0}}\left(B\left(x_{0}, \delta\right) \cap D\right)=B\left(x_{*}, \delta_{*}\right) \cap D_{*} .
$$

Definition 4.3. A family of domains $D_{h}\left(0<h \leq h_{0}\right)$ is said to be uniformly piecewise $C^{1,1}$ if in Definition $4.2 \delta$ can be chosen independently of $h$, and the diffeomorphism $G_{x_{0}} \equiv G_{x_{0}, h}$ have $C^{1,1}$ norms bounded independently of $h$.

Let $D$ be a piecewise $C^{1,1}$ bounded domain in $\mathbb{R}^{n} \quad(n \geq 2)$, and let $D_{h}$ be bounded domains in $\mathbb{R}^{n}$, uniformly piecewise $C^{1,1}$. Assume that $\partial D_{h}$ is given by

$$
\partial D_{h}: x=x_{0}+h \sigma_{h}\left(x_{0}\right) \nu\left(x_{0}\right)
$$

outside $\delta(h)$-neighborhood of the set $S$ of points of $\partial D$ where $\partial D$ is not $C^{1,1}$; here $\nu\left(x_{0}\right)$ is the outward normal, $\delta(h) \rightarrow 0$ if $h \rightarrow 0$, and

$$
\begin{gathered}
\left|\sigma_{h}\left(x_{0}\right)\right|+\left|\nabla_{x_{0}} \sigma_{h}\left(x_{0}\right)\right| \leq C, \\
\sigma_{h}\left(x_{0}\right) \rightarrow \sigma\left(x_{0}\right) \not \equiv 0 \text { as } h \rightarrow 0 .
\end{gathered}
$$

Remark 4.1. As shown in [1], if the $D_{h}$ are obtained from $D$ by affine transformations, then $(4.25)$ is valid. 
Theorem 4.4. Under the foregoing assumptions, if $D$ is strongly star-shaped with respect to the origin and $\left(\mathrm{A}_{4}\right)$ is satisfied then the stability property (4.2) holds.

The proof is similar to the proof of Theorems 4.1 and 4.2. In fact, once we can prove it for the case where $D, D_{h}$ are polyhedra, the proof for the general case follows by using the same estimates after performing local diffeomorphism about points of the set $S$.

In proving the theorem for polyhedra $D, D_{h}$, the main new effort is in extending Lemma 2.2 (upon which Lemma 3.1 depends). Here we can probably again apply eigenfunction expansion to $\zeta u$ where $\zeta$ is a cut-off function. We shall not attempt to carry it out since a proof of Lemma 2.2, which is valid in fact for any Lipschitz domain $D\left(f\right.$ is assumed to belong to $\left.L^{2 n /(n+1)}\right)$, was recently given by Escauriaza and Fabes [2]. We note however that Lemma 2.1 (used in the proof of Lemma 2.2) will be needed in $\S 6$; it is mainly for this reason that we have included in this paper our original proofs of Lemmas 2.1 and 2.2 .

We finally remark that if $n \geq 3$ we only need to use (3.8) for $\varepsilon=0$. Indeed, for $n=2$ (3.8) with $\varepsilon>0$ was used only in establishing (4.11). In the present case of $n \geq 3$, the trace theorem [7, p. 37] allows $p=2$ in (4.13), (4.14) and (4.15); thus (4.11) follows by using (3.8) with $\varepsilon=0$.

Remark 4.2. The star-shaped assumption on $D$ was used only in order to establish (4.22) for any $0<\lambda<1$. If $D$ is in $C^{1,1}$ then the star-shaped assumption may be dropped since $u^{i}(x)$ is in $H^{2}(D)$ and can therefore be extended into a function in $H^{2}\left(V_{\varepsilon}\right)$.

\section{THE NONMONOTONE CASE}

From now on we drop the monotonicity assumption (1.6) but assume that there exists a diffeomorphism $y=x+\phi_{h}(x)$ of $\Omega$ onto $\Omega$ which maps $D$ onto $D_{h}$ and satisfies

$$
\left|\nabla_{x} \phi_{h}(x)\right| \leq A h \quad(A \text { constant }) .
$$

Lemma 5.1. Let $D_{h}$ be uniformly piecewise $C^{1,1}$ domain and let $D$ be such that (4.24) and (4.25) hold. Assume also that (5.1) is satisfied. If the stability property (4.2) is not satisfied then

$$
k \int_{D} U \Delta v=(k-1) \int_{\partial D} \tilde{\sigma} \nabla \tilde{u} \cdot \nabla v \quad \forall v \in H^{2}(\Omega)
$$

holds, where

$$
\Delta \tilde{u}= \begin{cases}\nabla u^{e} & \text { if } \tilde{\sigma}(x)>0, \\ \nabla u^{i} & \text { if } \tilde{\sigma}(x)<0,\end{cases}
$$

and $\tilde{\sigma}(x)$ is a continuous function, $\tilde{\sigma}(x) \not \equiv 0$ and

$$
\operatorname{sgn} \tilde{\sigma}(x)=\operatorname{sgn} \sigma(x) .
$$

The proof is similar to the proof of (4.21) for polygonal domains in the monotone case; for $C^{1,1}$ domain the theorem was already proved in [1]. The main difference in the proof for the piecewise $C^{1,1}$ case occurs in establishing (4.19); it is here that the assumption (5.1) is needed (cf. [1], following the proof of Lemma 3.3). 
Corollary 5.2. If $\sigma(x)=0$ on a nonempty open subset of $\partial \Omega$ then the stability property (4.2) holds.

Indeed, this follows from the proof of Lemma 5.1 in precisely the same way as Corollary 3.4 of [1] which dealt with the case where $D$ and $D_{h}$ are $C^{1,1}$ domains.

In the remaining part of this section we assume that

$$
n=2 \text { and } \partial D \text { is analytic. }
$$

This implies that $u^{e}$ is analytic on $\partial D$. We shall prove that, for appropriately chosen $g$, the stability property (4.2) holds.

In addition to (5.5) we shall assume that

$$
\partial D \text { is strongly star-shaped with respect to the origin, }
$$

and that

$$
\sigma(x) \text { changes sign along } \partial D \text { only a finite number of times. }
$$

The assumption (5.6) is made so that one may apply (5.2) to a function as in (4.22) $(0<\lambda<1)$ and thus deduce, as $\lambda \uparrow 1$, that if the stability property (4.2) does not hold then

$$
\int_{\partial D} \tilde{\sigma} \nabla \tilde{u} \cdot \nabla v=0 \text { if } \Delta v=0 \text { in } D \text { and } \nabla v \in L^{1}(\partial D) .
$$

One can actually easily verify the condition (5.1) when (5.5) and (5.6) hold.

Assumption (5.7) implies that

$$
\{\tilde{\sigma}>0\}=\bigcup_{j=1}^{M} I_{j}^{+},
$$

$$
\{\tilde{\sigma}<0\}=\bigcup_{j=1}^{M^{\prime}} I_{j}^{-} \quad \text { where } I_{j}^{+}, I_{k}^{-} \text {are disjoint arcs on } \partial D \text {. }
$$

By (5.3),

$$
\nabla \tilde{u}= \begin{cases}\nabla u^{e} & \text { on } \bigcup_{j=1}^{M} I_{j}^{+}, \\ \nabla u^{i} & \text { on } \bigcup_{j=1}^{M^{\prime}} I_{j}^{-}\end{cases}
$$

In view of Corollary 5.2, we may assume from now on that $M^{\prime}=M$ and the union of the $\overline{I_{j}^{+}}, \overline{I_{k}^{-}}$is all of $\partial D$.

Lemma 5.3. If for any $C^{1}$ function $h$ there exists a solution $w$ to

$$
\begin{aligned}
\Delta w & =0 \quad \text { in } D, \\
\nabla \tilde{u} \cdot \nabla w & =h \quad \text { on } \partial D, \\
\nabla w & \in L^{1}(\partial D),
\end{aligned}
$$

then the stability property (4.2) holds.

Indeed, using (5.8) we conclude that

$$
\int_{\partial D} \tilde{\sigma} h=0
$$

since $h$ is arbitrary, $\tilde{\sigma}=0$ which is a contradiction. 
Remark 5.1. It is actually sufficient to solve (5.11) just for $h=\tilde{\sigma}$, but $\tilde{\sigma}$ may not be $C^{1}$.

In order to establish (5.11) we shall rely on the index theory for the RiemannHilbert problem as exposed in [8].

We recall (see $[8, \S 40,(40.8)]$ ) that for a continuous vector field $V=a+i b$ on $\bar{\Omega}$ and a smooth curve $\Gamma \subset \bar{\Omega}$ which is the boundary of a subdomain in $\Omega$ one defines the index of $V$ with respect to $\Gamma$ by

$$
\kappa(V ; \Gamma)=\frac{1}{\pi}[\arg (a-i b)]_{\Gamma}
$$

provided $V \neq 0$ on $\Gamma$.

It is well known that the index is homotopic invariant, i.e., if $V(\theta)$ is a family of such vector fields continuous in $\theta, 0 \leq \theta \leq 1$, then

$$
\kappa(V(0) ; \Gamma)=\kappa(V(1) ; \Gamma)
$$

provided $V(\theta) \neq 0$ on $\Gamma$ for all $\theta$. The definition of the index of $V=a+i b$ with respect to $\Gamma$ can be extended to the case where $V$ may vanish or have finite number of jump-discontinuities at points $c_{1}, \ldots, c_{N}$ on $\Gamma$. Setting $G=$ $(a-i b) /(a+i b)$ one defines (see $[8, \S 93$, p. 273]),

$$
\kappa(V ; \Gamma)=\frac{1}{2 \pi}[\arg G]_{\Gamma}
$$

provided the limits $G\left(c_{i} \pm 0\right)$ exist, where the passage from $G\left(c_{i}+0\right)$ to $G\left(c_{i}-0\right)$ is selected as [8, §85]. If $V(\theta)$ varies continuously with $\theta$ and each $V(\theta)$ vanishes or has jump-discontinuities only at $c_{1}, \ldots, c_{N}$ then $(5.13)$ is still valid provided the limits $G\left(c_{i} \pm 0\right)$ exist for all $0 \leq \theta \leq 1$.

Consider the example of

$$
V_{0}(\theta)=\left(1-\frac{(k-1) \theta}{k}\right) u_{N}^{e} \vec{N}+u_{\tau}^{e} \vec{\tau} \quad(0 \leq \theta \leq 1)
$$

where $\vec{N}$ is the outward unit normal and $\vec{\tau}$ is unit tangent (in the counterclockwise direction) along $\partial D$. From the diffraction conditions

$$
u^{e}=u^{i}, \quad \frac{\partial u^{e}}{\partial N}=k \frac{\partial u^{i}}{\partial N}
$$

we see that, for any $z \in \partial D$,

$$
\nabla u^{e}(z) \neq 0 \text { if and only if } \nabla u^{i}(z) \neq 0 \text {. }
$$

Since $V_{0}(\theta)$ is a homotopy from $\nabla u^{e}$ to $\nabla u^{i}$, we conclude that

$$
\left.\kappa\left(\nabla u^{e} ; \partial D\right)=\kappa\left(\nabla u^{i} ; \partial D\right)\right) \quad \text { if } \nabla u^{e} \neq 0 \text { on } \partial D \text {. }
$$

Consider next the vector field

$$
V_{1}(\theta)= \begin{cases}V_{0}(\theta) & \text { on } \partial D^{+} \equiv \bigcup_{j} I_{j}^{+} \\ \nabla u^{i} & \text { on } \partial D^{-} \equiv \bigcup_{j} I_{j}^{-}\end{cases}
$$

Clearly $V_{1}(0)=\nabla \tilde{u}$ and $V_{1}(1)=\nabla u^{i}$. Notice that $V_{1}(\theta)$ has a finite number of jump discontinuities along $\partial D$, i.e., at the endpoints of the $I_{j}^{-}$. As explained above the invariance formula $(5.13)$ is still valid, so that

$$
\kappa\left(\nabla u^{i} ; \partial D\right)=\kappa(\nabla \tilde{u} ; \partial D) \text { provided } \nabla u^{i} \neq 0 \text { on } \partial D \text {. }
$$


The Riemann-Hilbert problem in $D$ is concerned with finding a holomorphic function $\phi$ in $D$, continuous in $\bar{D}$, such that

$$
a \operatorname{Re} \phi+b \operatorname{Im} \phi=c \text { on } \partial D
$$

here $D$ is a $C^{1}$ domain and $a, b, c$ are piecewise continuous with a finite number of discontinuities $z_{1}, \ldots, z_{N}$, and their derivatives are bounded in each arc $z_{j} z_{j+1} \quad\left(z_{N+1}=z_{1}\right)$. By [8, §93], if $a^{2}+b^{2}>0$ and the index of $V=a+i b$ with respect to $\partial D$ is $\geq-1$ then for any $c$ there exists a solution $\phi$ and $[8,(93.1)]$,

for $z$ near $z_{j}$.

$$
|\phi(z)| \leq \frac{C}{\left|z-z_{j}\right|^{\alpha}} \quad(0<\alpha<1)
$$

We note that the Riemann-Hilbert problem for holomorphic function $\phi(z)$ is equivalent to the problem

$$
\begin{aligned}
& \Delta v=0 \text { in } D, \\
& a v_{x}+b v_{y}=c \text { on } \partial D \text {, }
\end{aligned}
$$

for $v=\operatorname{Re} \int \phi(z) d z$. We therefore conclude:

Lemma 5.4. If $\kappa(\nabla \tilde{u} ; \partial D) \geq-1$ then for any piecewise $C^{1}$ function $h$ there exists a solution to (5.11).

The fact that $\nabla w \in L^{1}(\partial D)$ follows from the estimate of $\phi(z)$ near $z_{j}$, where $z_{j}$ are the points of discontinuity of $V$. Recalling (5.18) we have thus reduced the proof of the stability property $(4.2)$ to showing that

$$
\nabla u^{i} \neq 0 \text { on } \partial D
$$

and

$$
\kappa\left(\nabla u^{i} ; \partial D\right) \geq-1 .
$$

Since $u^{i}$ is analytic in $\bar{D}$, it has analytic extension into a neighborhood $N^{+}$ of $\bar{D}$; we denote it by $u^{i}$ and note that $u^{i}$ is harmonic in $N^{+}$. Similarly $u^{e}$ has analytic (and harmonic) extension into an $\Omega$-neighborhood $N^{-}$of $\Omega \backslash D$.

We shall now make a special choice of $g$ as follows:

Definition of $g$. Let $z=z(t)$ be a $C^{1, \alpha}$ parametrization of $\partial \Omega(0 \leq t \leq 2 \pi)$ and let $f(z(t))$ be a $C^{1, \alpha}$ function such that $f(z(t))$ has a unique maximum at $t=0$, a unique minimum at some point $t=t_{0}$, and

$$
\begin{array}{ll}
\frac{d}{d t} f(z(t))<0 & \text { if } 0<t<t_{0}, \\
\frac{d}{d t} f(z(t))>0 & \text { if } t_{0}<t<2 \pi .
\end{array}
$$

Let $u$ be the solution of the diffraction problem

$$
\begin{aligned}
\operatorname{div}(a \nabla u)=0 & \text { in } \Omega, \\
u=f & \text { on } \partial \Omega,
\end{aligned}
$$

and set

$$
g=\frac{\partial u}{\partial \nu}
$$

We shall prove 
Lemma 5.5. For the special choice of $g$ in (5.23), (5.19) holds and

$$
\kappa\left(\nabla u^{i} ; \partial D\right)=0 \text {. }
$$

Since (5.24) implies (5.20), we deduce

Theorem 5.6. Under the assumptions (5.5)-(5.7), the stability property (4.2) holds.

Proof of Lemma 5.5. From the transmission conditions (5.16) one can easily show that $u$ cannot take minimum or maximum at points on $\partial D$. Therefore $u$ attains its maximum at $z(0)$ and its minimum at $z\left(t_{0}\right)$ and, by the maximum principle, $\partial u / \partial \nu \neq 0$ at these two points. At all other points of $\partial \Omega$ we also have $\partial u / \partial \tau \neq 0$ (by the choice of $f$ ). Consequently $\nabla u^{e} \neq 0$ on $\partial \Omega$. Since further the tangential components of $\nabla u^{e}$ have the same sign on $\left(0, t_{0}\right)$ and (the reverse sign) on $\left(t_{0}, 2 \pi\right)$, it can be seen that

$$
\kappa\left(\nabla u^{e} ; \partial \Omega\right)=0 .
$$

The vector field $\nabla u^{i}$ has a finite number of zeros $z_{1}, \ldots, z_{m}$ in $\bar{D}$ and similarly (since $\nabla u^{e} \neq 0$ in an $\Omega$-neighborhood of $\partial \Omega$ ) the vector field $\nabla u^{e}$ has a finite number of zeros in $z_{m+1}, \ldots, z_{\sigma}$ in $\Omega \backslash D$. On $\partial D, \nabla u^{i}$ and $\nabla u^{e}$ have common zeros (if any); we denote them by $z_{l+1}, z_{l+2}, \ldots, z_{m}$.

Let $L_{\varepsilon}$ be the Jordan curve formed by the arcs of the $\partial B\left(z_{j} ; \varepsilon\right) \quad(l+1 \leq$ $j \leq m)$ which are contained in $\Omega \backslash D$ and by $\partial D \backslash \bigcup_{j=l+1}^{m} B\left(z_{j} ; \varepsilon\right)$. We claim

Lemma 5.7. If $\varepsilon$ is sufficiently small then

$$
\left.\nabla u^{e}\right|_{L_{\varepsilon}} \text { is homotopic to }\left.\nabla u^{i}\right|_{L_{\varepsilon}} \text {. }
$$

Proof. If $\varepsilon$ is sufficiently small then $\nabla u \neq 0$ in a neighborhood of

$$
\Gamma_{\varepsilon} \equiv \partial D \backslash \bigcup_{l+1}^{m} B\left(z_{j} ; \varepsilon\right) .
$$

Let $\nabla u^{e}=u_{N}^{e} \vec{N}+u_{\tau}^{e} \vec{\tau}$. Then $u_{N}^{e}$ and $u_{z}^{e}$ do not vanish simultaneously on $\Gamma_{\varepsilon}$. We define, for $0 \leq \theta \leq 1$,

$$
V(\theta)=\left(1-\frac{k-1}{k} \theta\right) u_{N}^{e} \vec{N}+u_{\tau}^{e} \vec{\tau} \text { on } \Gamma_{\varepsilon} .
$$

Then $V(0)=\nabla u^{e}, V(1)=\nabla u^{i}$ and $V(\theta)$ is continuous in $\theta$; moreover, $V(\theta) \neq 0$ on $\bar{\Gamma}_{\varepsilon}$.

We next wish to define $V(\theta)$ on any arc $\partial B\left(z_{j} ; \varepsilon\right) \backslash \bar{D}$ of $L_{\varepsilon}$. To do this we introduce a conformal mapping of the lower half-plane onto $D$ which maps 0 into $z_{j}$. By analytic continuation, the mapping is conformal in a neighborhood of 0 . Since the refraction conditions (5.16) are invariant under conformal mapping, we may assume from the start that $z=0$ and that $D$, near $z=0$, coincides with the lower half-plane. Expanding $u^{e}, u^{i}$ near $z=0$ into series

$$
\begin{aligned}
u^{e} & =\sum r^{n}\left(a_{n}^{e} \cos n \varphi+b_{n}^{e} \sin n \varphi\right), \\
u^{i} & =\sum r^{n}\left(a_{n}^{i} \cos n \varphi+b_{n}^{i} \sin n \varphi\right)
\end{aligned}
$$

and using the refraction conditions, we obtain

$$
a_{n}^{e}=a_{n}^{i}, \quad b_{n}^{e}=k b_{n}^{i} .
$$


We now define (in the variables $(r, \varphi)$ of the conformal mapping) $V(\theta)=$ $\nabla u(\theta)$ where

$$
u(\theta)=\sum r^{n}\left(a_{n}^{e} \cos n \varphi+\left(1-\frac{k-1}{k} \theta\right) b_{n}^{e} \sin n \varphi\right) .
$$

Then, by $(5.28), u(0)=u^{e}, u(1)=u^{i}$ so that

$$
V(0)=\nabla u^{e}, \quad V(1)=\nabla u^{i} .
$$

Further,

$$
\nabla V(\theta) \neq 0 \quad \text { on } \partial B\left(z_{j} ; \varepsilon\right)
$$

and $V(\theta)$ continuously fits with $(5.27)$ at the two points of $\partial \Gamma_{\varepsilon} \cap \partial B\left(z_{j} ; \varepsilon\right)$.

We have thus constructed a homotopy $V(\theta)$ of $\nabla u^{i}$ along $L_{\varepsilon}$; this establishes the assertion (5.26).

Completion of the proof of Lemma 5.5. Consider the index

$$
\kappa(\zeta) \equiv \kappa(\nabla u ; \partial B(\zeta ; \varepsilon)) \quad(\varepsilon \text { small })
$$

of $\nabla u$ at a zero $k=\zeta$ of $\nabla u$, where $\zeta \in \Omega \backslash \partial D$. Introducing $h=u+i v \quad(h$ holomorphic), we have

$$
h^{\prime}(z)=u_{x}+i v_{x}=u_{x}-i u_{y},
$$

and

$$
\begin{aligned}
\pi \kappa(\zeta) & =\pi \kappa\left(u_{x}-i u_{y} ; \partial B(\zeta ; \varepsilon)\right)=\operatorname{Var}_{\partial B(\zeta ; \varepsilon)} h^{\prime}(z) \\
& =\operatorname{Var}_{\partial B(\zeta ; \varepsilon)}(z-\zeta)^{n-1}=2 \pi(n-1)
\end{aligned}
$$

where $h(z)=a_{0}(z-\zeta)^{n}+\cdots, a_{0} \neq 0$. Hence

$$
\kappa(\zeta)=2 \times\{\text { order of zero of } \nabla u \text { at } z=\zeta\} .
$$

Denote by $\pi_{j}$ the order of the zero of $\nabla u$ at $z=z_{j}$. Then, for small $\varepsilon>0$,

$$
\kappa\left(\nabla u^{e} ; \partial \Omega\right)=\kappa\left(\nabla u^{e} ; L_{\varepsilon}\right)+\sum_{j=m+1}^{\sigma} 2 \pi_{j} .
$$

From Lemma 5.7 we also have

$$
\kappa\left(\nabla u^{e} ; L_{\varepsilon}\right)=\kappa\left(\nabla u^{i} ; L_{\varepsilon}\right) .
$$

Finally, by (5.29),

$$
\kappa\left(\nabla u^{i} ; L_{\varepsilon}\right)=\sum_{j=1}^{m} 2 \pi_{j} .
$$

Combining (5.30)-(5.32) and recalling (5.25) we deduce that

$$
\sum_{j=1}^{\sigma} \pi_{j}=0 .
$$

Hence $\nabla u$ has no zeros in $\bar{D}$ and (5.24) holds. This completes the proof of Lemma 5.5 and therefore also of Theorem 5.6.

\section{The NONMONOTONE CASE WITH PIECEWISE ANALYTIC $\partial D$}

In this section we continue to consider the nonmonotone case for $n=2$, but assume that $\partial D$ is piecewise analytic with a finite number of vertices 
$s_{1}, \ldots, s_{N}$. For simplicity we take $D$ and $D_{h}$ to be polygons, as in $\S 2$, with $D$ convex, and

$$
c_{1} h \leq \sum_{j=1}^{N}\left|s_{j}(h)-s_{j}\right| \leq c_{2} h \quad\left(0<c_{1}<c_{2}<\infty\right) ;
$$

however our results easily extend to any piecewise analytic $\partial D$.

Our starting point is Lemma 5.1; as in the proof of Theorem 5.6 (recall Remark 5.1) the stability property (4.2) holds if there exists a solution $v$ to

$$
\begin{aligned}
& \Delta v=0 \text { in } D, \\
& \nabla \tilde{u} \cdot \nabla v=\tilde{\sigma} \quad \text { on } \partial D \text {, }
\end{aligned}
$$

such that $\nabla v_{\lambda} \rightarrow \nabla v$ in $L^{1}(\partial D)$ as $\lambda \uparrow 1 ; v_{\lambda}(x)=v\left(x_{0}+\lambda\left(x-x_{0}\right)\right)$ for some $x_{0} \in D$. The function $\tilde{\sigma}$ is linear on each edge $\Gamma_{j}$.

By Lemma 2.1 it follows that any $\nabla \tilde{u}$ has a finite variation along any arc of $\partial D$ which contains a vertex $s_{j}$ of $\partial D_{i}$; consequently

$$
\kappa(\nabla \tilde{u} ; \partial D)<\infty \text {. }
$$

In the original theory of Muskhelishvili $[8, \S \S 93,94]$ the domain $D$ is assumed to be smooth. However, the results remain true if $\partial D$ is piecewise $C^{1}$; see [3, Example 8.4]. One can see it by using conformal mapping $z=z(\omega)$ of the unit disc $\{|\omega|<1\}$ onto $D$, and applying the original theory in the $\omega$-domain noting that the index of $z^{\prime}(\omega)$ is zero since $\omega \rightarrow z(\omega)$ is conformal (i.e. $z^{\prime}(\omega)$ does not vanish in the unit disc).

If $\kappa \geq-1$ then (by [8, §93]) there exists a unique solution of $(6.1),(6.2)$ satisfying

$$
|\nabla v| \leq \frac{C}{\left|x-c_{j}\right|^{\alpha}} .
$$

On the other hand, if $\kappa \leq-2$ then there are $-\kappa-1$ solutions of the homogeneous problem, and the solution $v$ of $(6.1),(6.2),(6.4)$ exists if and only if $\tilde{\sigma}$ is orthogonal to these solutions. The orthogonality relations can be written in the form

$$
\int_{\partial D} \tilde{\sigma} l_{m}=0, \quad m=1, \ldots,-\kappa-1 .
$$

We summarize

Theorem 6.1. (i) If $\kappa \geq-1$ then the stability property (4.2) holds;

(ii) if $\kappa \leq-2$ and

$$
\tilde{\sigma} \text { is not a linear combination of } l_{1}, \ldots, l_{-\kappa-1}
$$

then the stability property (4.2) holds.

This result for analytic $\partial D$ was proved in [1]. $\S 5$.

We now wish to estimate the index $\kappa$ for the special choice of $g$ made in

From the results of $[9$, p. 201] it follows that the solution $u$ of the diffraction problem (3.2) cannot take local maximum (or local minimum) at a vertex of $\partial D$. So as in the proof of $(5.25)$ : 
Lemma 6.2. For the special choice of $g$ in (5.21)-(5.23),

$$
\kappa\left(\nabla u^{e} ; \partial \Omega\right)=0 \text {. }
$$

From now on we shall work with the special choice of $g$ in (5.21)-(5.23).

Lemma 6.3. $\nabla u(x) \neq 0$ for $x \neq s_{1}, \ldots, s_{N}$.

Proof. Let $\xi_{j}, \eta_{j}$ be points on $\partial D, \xi_{j} \in \Gamma_{j}$ and $\eta_{j} \in \Gamma_{j+1}$, such that $\left|\xi_{j}-s_{j}\right|$ and $\left|\eta_{j}-s_{j}\right|$ are small and $\nabla u^{e}\left(\xi_{j}\right) \neq 0 \nabla u^{e}\left(\eta_{j}\right) \neq 0$. Let $\widetilde{\Gamma}_{\varepsilon}$ be $C^{2}$ and piecewise analytic curves in $\bar{D}$ which converge to $\partial D$ as $\varepsilon \rightarrow 0$ such that $\widetilde{\Gamma}_{\varepsilon}$ connects $\xi_{j}$ to $\eta_{j}$ by an analytic arc and it is a line-segment between $\eta_{j}$ and $\xi_{j+1}$ (this segment lies on $\partial D$ ). Denote by $\widetilde{D}_{\varepsilon}$ the domain bounded by $\widetilde{\Gamma}_{\varepsilon}$, and let $\tilde{u}_{\varepsilon}$ denote the solution of the refraction problem corresponding to $\tilde{\Gamma}_{\varepsilon}$. Then

$$
\begin{aligned}
& \nabla \tilde{u}_{\varepsilon}^{i} \rightarrow \nabla u^{i} \text { and } \nabla \tilde{u}_{\varepsilon}^{e} \rightarrow \nabla u^{e} \text { uniformly outside any neighbor- } \\
& \text { hood of }\left\{s_{1}, \ldots, s_{N}\right\} .
\end{aligned}
$$

Observe that $\nabla \tilde{u}_{\varepsilon} \neq 0$ at $\xi_{j}, \eta_{j}$ for all $\varepsilon$, and that $\nabla \tilde{u}_{\varepsilon}^{e}, \nabla \tilde{u}_{\varepsilon}^{i}$ are analytic across $\widetilde{\Gamma}_{\varepsilon} \backslash\left\{\xi_{1}, \eta_{1}, \ldots, \xi_{N}, \eta_{N}\right\}$. Therefore $\nabla \tilde{u}_{\varepsilon}^{e}$ and $\nabla \tilde{u}_{\varepsilon}^{i}$ have only a finite number of zeros. Since also $\kappa\left(\nabla \tilde{u}_{\varepsilon} ; \partial \Omega\right)=0$, we can repeat an argument used in $\S 5$ and deduce that

$$
\begin{array}{ll}
\nabla \tilde{u}_{\varepsilon}^{e}(x) \neq 0 & \text { in } \bar{\Omega} \backslash \widetilde{\Gamma}_{\varepsilon}, \\
\nabla \tilde{u}_{\varepsilon}^{i}(x) \neq 0 & \text { in } \widetilde{D}_{\varepsilon} \cup \widetilde{\Gamma}_{\varepsilon} .
\end{array}
$$

We now suppose that $\nabla u^{e}\left(x_{0}\right)=0$ for some $x_{0} \in \Omega \backslash D, x_{0} \neq$ vertex. If $x_{0} \in \partial D$ then we choose the $\xi_{j}, \eta_{j}$ above so that $x_{0}$ lies on one of the line segments of $\tilde{\Gamma}_{\varepsilon}$. Then (whether $x_{0} \in \partial D$ or $x_{0} \notin \partial D$ ) there exists a small disc $B_{\delta}\left(x_{0}\right)$ such that $u^{e}$ and $\tilde{u}_{\varepsilon}^{e}$ are analytic in $\overline{B_{\delta}\left(x_{0}\right)}$ and $\nabla u^{e} \neq 0$ on $\partial B_{\delta}\left(x_{0}\right)$. From (6.8), (6.9) we then deduce that

$$
0=\kappa\left(\nabla \tilde{u}_{\varepsilon}^{e} ; x_{0}\right)=\kappa\left(\nabla \tilde{u}_{\varepsilon}^{e} ; \partial B_{\delta}\left(x_{0}\right)\right)=\kappa\left(\nabla u^{e} ; \partial B_{\delta}\left(x_{0}\right)\right)=\kappa\left(\nabla u^{e} ; x_{0}\right)
$$

if $\varepsilon$ is small enough, which is a contradiction.

Similarly one can prove that $\nabla u^{i}(x) \neq 0$ if $x \in \bar{D}, x \neq$ vertex.

Denote by $\widetilde{\Gamma}_{\varepsilon}^{e}$ smooth curves in $\Omega \backslash D$ such that $\widetilde{\Gamma}_{\varepsilon}^{e}$ coincides with $\partial D$ outside $\varepsilon$-neighborhood of each $s_{j}$, and such that $\widetilde{\Gamma}_{\varepsilon}^{e}$ connects a point in $\Gamma_{j}$ to a point in $\Gamma_{j+1}$ by an arc $\sigma_{j, \varepsilon}^{e}$ which "approximately" lies on $\left|z-s_{j}\right|=\varepsilon$; by "approximately" we mean that we make $\widetilde{\Gamma}_{\varepsilon}^{e}$ smooth as it intersects $\partial D$, by slightly modifying the arc $\left|z-s_{j}\right|=\varepsilon$. Similarly we define curves $\widetilde{\Gamma}_{\varepsilon}^{i}$ and the approximate $\operatorname{arcs} \sigma_{j, \varepsilon}^{i}$ in $D$.

By Lemma 2.1, near $s_{j}$,

$$
\begin{aligned}
& \overline{\nabla u^{e}}=A_{j}^{e}\left(z-s_{j}\right)^{\gamma_{j}-1}(1+o(1)), \\
& \overline{\nabla u^{i}}=A_{j}^{i}\left(z-s_{j}\right)^{\gamma_{j}-1}(1+o(1)),
\end{aligned}
$$

where $A_{j}^{e} \neq 0, A_{j}^{i} \neq 0$ and $\gamma_{j}$ is a positive number larger than $1 / 2$. Indeed, take for simplicity $j=1, s_{j}=0$. By (2.21),

$$
u=\operatorname{Re} \sum_{m=1}^{k-1} B_{m} z^{\gamma_{m}}+\sum_{m=k}^{\infty} C_{m} r^{\gamma_{m}} v_{m}(\theta), \quad B_{1} \neq 0 ;
$$


$k$ is chosen so that $k>1$ and $\gamma_{k}>3+\gamma_{1}$. By (2.13) and (2.11)

$$
\int_{0}^{2 \pi}\left(v_{m}^{\prime}(\theta)\right)^{2} \leq C \gamma_{m}^{2}
$$

so that

$$
\left|v_{m}(\theta)\right| \leq C \gamma_{m} .
$$

Therefore, by (2.11),

$$
\left|v_{m}^{\prime \prime}(\theta)\right| \leq C \gamma_{m}^{3} \quad \text { if } \theta \neq 0, \quad \theta \neq \beta
$$

and then also

$$
\left|v_{m}^{\prime}(\theta)\right| \leq C \gamma_{m}^{3} .
$$

Using (6.12), (6.13) and (2.22), (2.7), we deduce from (6.11) that

$$
\overline{\nabla u}=\operatorname{Re} \sum_{m=1}^{k-1} \gamma_{m} B_{m} z^{\gamma_{m}-1}+O\left(r^{\gamma_{k}-3}\right),
$$

which implies (6.10).

From Lemma 6.4 and Corollary 6.3 we have

$$
\begin{gathered}
\kappa\left(\nabla u^{e} ; \widetilde{\Gamma}_{\varepsilon}^{e}\right)=0, \\
\kappa\left(\nabla u^{i} ; \widetilde{\Gamma}_{\varepsilon}^{i}\right)=0 .
\end{gathered}
$$

Take any vertex $s_{j}$ and introduce polar coordinates $(r, \theta)$ about $s_{j}$. We want to evaluate the index of $\nabla u^{i}$ with respect to the boundary of a small domain $\Omega_{j} \subset D$ such that $\partial \Omega_{j}$ consists of two line segments

$$
l_{1}=\left\{(r, 0), 0 \leq r \leq \delta_{0}\right\}, \quad l_{2}=\left\{\left(r, \beta_{j}\right), 0 \leq r \leq \delta_{0}\right\}
$$

and a circular arc

$$
l_{3}=\left\{\left(\delta_{0}, \theta\right), 0 \leq \theta \leq \beta_{j}\right\} .
$$

Set $\Sigma_{\varepsilon}=l_{2} \cup l_{1} \cup l_{3}$. By the definition of index in (5.14) and the expansion (6.10) for $\nabla u^{i}$,

$$
\begin{aligned}
\kappa\left(\nabla u^{i} ; \Sigma_{\varepsilon}\right) & =\frac{1}{2 \pi} \arg \left[\frac{\overline{\nabla u^{i}}}{\nabla u^{i}}\right]_{l_{3}}+\frac{1}{2 \pi} \arg \left[\overline{\frac{\nabla u^{i}}{\nabla u^{i}}}\right]_{l_{2} \cup l_{1}} \\
& =\frac{\left(\gamma_{j}-1\right) \beta_{j}}{\pi}+\frac{1}{\pi}\left\{\operatorname{jump~of~} \arg \left(z-s_{j}\right)^{\gamma_{j}-1}\right. \\
& \text { from } \left.\theta=\beta_{j} \text { to } \theta=0 \text { at } r=0\right\} \\
& =\frac{\left(\gamma_{j}-1\right) \beta_{j}}{\pi}-\left\{\text { nonintegral part of } \frac{\left(\gamma_{j}-1\right) \beta_{j}}{\pi}\right\} \\
& =\left[\frac{\left(\gamma_{j}-1\right) \beta_{j}}{\pi}\right]
\end{aligned}
$$

where $[x]$ is the integral part of $x$ if $x>0$ and $[x]=0$ if $x<0$ (if $\gamma_{j}-1<0$ then, since $\gamma_{j}>\frac{1}{2}$, the right-hand side of (6.16) is nonpositive and smaller than 1 in absolute value; since the index is an integer, it must then be equal to zero).

Repeating the above argument at each vertex $s_{j}$ and recalling (6.15) we deduce 
Lemma 6.5. $\kappa\left(\nabla u^{i} ; \partial D\right) \geq 0$.

To compute $\kappa(\nabla \tilde{u} ; \partial D)$ we deform $\nabla u^{i}$ into $\nabla \tilde{u}$. Consider first the case where $\nabla \tilde{u}$ is obtained by replacing $\nabla u^{i}$ by $\nabla u^{e}$ on a single closed arc $\sigma$. If $\sigma$ lies inside one edge $\Gamma_{j}$ then

$$
\kappa\left(\nabla u^{i} ; \partial D\right)-\kappa(\nabla \tilde{u} ; \partial D)=\frac{\lambda_{1}}{\pi}+\frac{\lambda_{2}}{\pi}
$$

where $\lambda_{k}$ is the difference in the arguments of $\nabla u^{i}$ and $\nabla u^{e}$ at an endpoint of $\sigma$. Since the index is an integer whereas $\left|\lambda_{k}\right|<\pi / 2$, it follows that

$$
\kappa\left(\nabla u^{i} ; \partial D\right)-\kappa(\nabla \tilde{u} ; \partial D)=0 .
$$

Suppose next that $\sigma$ contains a vertex $s_{j}$ and its endpoints lie inside $\Gamma_{j}$ and $\Gamma_{j+1}$. Then, by (5.14),

$$
\kappa\left(\nabla u^{i} ; \partial D\right)-\kappa(\nabla \tilde{u} ; \partial D)=\frac{\lambda_{1}}{\pi}+\frac{\lambda_{2}}{\pi}+\frac{\Delta}{2 \pi}
$$

where $\Delta$ is the difference between

$$
B^{i} e^{2 \sqrt{-1}\left(\gamma_{j}-1\right) \beta_{j}}-B^{i} e^{2 \sqrt{-1}\left(\gamma_{j}-1\right) 0} \quad\left(B^{i}=\frac{\alpha_{i}-\sqrt{-1} \beta_{i}}{\alpha_{i}+\sqrt{-1} \beta_{i}}, \alpha_{i}+\sqrt{-1} \beta_{i}=A_{j}^{i}\right)
$$

and the corresponding expression with $B^{e}$ (associated with $A_{j}^{e}$ ). Since the index is an integer and $\left|\left(\lambda_{1}+\lambda_{2}\right) / \pi\right|<1$, there is no ambiguity about the choice of the corresponding limits $G\left(s_{j} \pm 0\right)$ if $\left|A^{i}-A^{e}\right|$ is small; in fact, the correct choice is such that the limits of

$$
\frac{1}{B^{i}} \frac{\bar{\nabla} u^{i}}{\nabla u^{i}} \text { and } \frac{1}{B^{e}} \frac{\overline{\nabla u^{e}}}{\nabla u^{e}} \text { agree at } s_{j} \pm 0
$$

so that the left-hand side of $(6.18)$ is equal to zero. By continuously deforming $A^{e}$ we deduce that (6.17) holds in the general case.

Finally, a similar argument shows that (6.17) holds if $\sigma$ lies in $\Gamma_{j}$ and one of its endpoints is a vertex of $\partial D$.

By deforming $\nabla u^{i}$ step-by-step a finite number of times so as to obtain $\nabla \tilde{u}$, and applying (6.17) at each step, we deduce that (6.17) is valid for general $\nabla \tilde{u}$. Consequently, by Lemma 6.5 ,

$$
\kappa(\nabla \tilde{u} ; \partial D) \geq 0 .
$$

This together with Theorem 6.1 implies

Theorem 6.6. For the special choice of $g$ made in (5.21)-(5.23), the stability property (4.2) holds.

\section{REFERENCES}

1. H. Bellout and A. Friedman, Identification problems in potential theory, Arch. Rational Mech. Anal. 101 (1988), 143-160.

2. L. Escauriaza, E. Fabes, and G. Verchota, On a regularity theorem for weak solutions to transmission problems with internal Lipschitz boundary, Proc. Amer. Math. Soc. (to appear).

3. G. Eskin, Index formulas for elliptic boundary value problems in plane domains with corners, Trans. Amer. Math. Soc. 314 (1989), 283-348.

4. A. Friedman, Partial differential equations, Holt, Rinehart and Winston, New York, 1969 (reprinted by Krieger). 
5. A. Friedman and B. Gustafsson, Identification of the conductivity coefficient in an elliptic equation, SIAM J. Math. Anal. 18 (1987), 777-787.

6. A. Friedman and V. Isakov, On the uniqueness in the inverse conductivity problem with one measurement, Indiana Univ. Math. J. 38 (1989), 553-580.

7. P. Grisvard, Elliptic problems in nonsmooth domains, Pitman, London, 1985.

8. N. I. Muskhelishvili, Singular integral equations, Noordhoff, Groningen, 1953.

9. G. Stampacchia, Équations elliptiques du second ordre à coefficients discontinues, Lecture Notes, Univ. de Montreal, 1965.

Department of Mathematics, Northern Illinois University, De Kalb, Illinois 60115

Institute for Mathematics and its ApPlications, University of Minnesota, MinNeapoLis, Minnesota 55455

E-mail address: bellout@math.niu.edu

Department of Mathematics, Wichita State University, Wichita, Kansas 67208 